\title{
3D reconstruction of the operating field for image overlay in 3D-endoscopic surgery. ${ }^{*}$
}

\author{
Fabien Mourgues, Frédéric Devernay, Ève Coste-Manière \\ CHIR Medical Robotics Team www.inria.fr/chir, \\ InRIA, BP93, 06902 Sophia Antipolis Cedex, France
}

\begin{abstract}
Building a 3D model of the operating field is the first stage to access the registration of pre-operative surface models in per-operative context. We propose a method to learn the 3D model of the organs observed by a stereoscopic endoscope. This method is general and requires no a priori knowledge of the position of the instruments, whether robotized or manually-controlled, present in the field of view. The result of the learning process allows the removal of the instruments from the surgeon's field of view: "diminished reality". This work prefigures the $3 D$ integration by augmented reality of preoperative organs model (e.g. arteries) in stereoscopic images. Experiments are currently being performed to validate our approach using endoscopic sequences from the da Vinci $^{T M}$ surgical system.
\end{abstract}

\section{Introduction}

While totally endoscopic surgery limits trauma and reduces post-operative recovery times, it provides the surgeon a limited keyhole view of the operating field delivered by the endoscope. This confined vision greatly increases the difficulty of locating landmarks necessary for the operation; e.g. a target artery hidden by fat or the position of a stenosis along an artery in cardiac surgery [6].

As described in [3], we propose to enhance the surgeon's endoscopic vision with overlaid cartographic information indicating the position of the targets with respect to the field of view [1]. The available augmented reality systems based on 3D laparoscopy [2, 5] use 3D position sensors and are not designed for robotically-assisted surgery.

Because of the displacement of organs during operation, the problem of registration in the operating room can not be solved solely by using a priori knowledge of the position of the endoscope with respect to the patient. Hence the overall proposed approach consists in registering the 3D surface model of the organs observed by the stereoscopic endoscope with a pre-operative surface model; for example, in heart

\footnotetext{
* Thanks to the following persons and institutions for providing data for our experiments and feedback: Alain Carpentier, Renaud Séverac-Bastide, Didier Blanchard, Hôpital Européen Georges Pompidou; Kenneth Salisbury, Gary Guthart, Intuitive Surgical.
}

surgery a time variant model of the heart obtained by the fusion of preoperative information extracted from standard angiographic sequences [7] and MRI or CT-scan data.

In this paper we only describe the initial step of the overall process which consists in building a 3D surface model of the observed organs. For one endoscopic position, the 3D surface of the organ is learned by separating the instruments moving in the foreground of the observed scene. A global 3D surface model can be obtained by exploring the surgical volume with a newly adapted protocol. After the learning stage is completed, the instruments can be removed from the endoscopic images ("diminished reality") by foreground/background separation.

\section{Material and methods}

Even if the tools are robotized, other manuallycontrolled instruments are present in the operating field. Our method is general and does not use any prior information regarding the position of the instruments.

The per-operative images are acquired with the stereoscopic endoscope delivering the 3D vision of the operating field to the surgeon. The endoscope is calibrated with a planar calibration grid observed from different points of view [8].

\subsection{Stereoscopy}

First, the endoscopic images are rectified and a correlation-based stereo algorithm is used to obtain a 3D reconstruction of the scene in the frame of the endoscope. This reconstruction - the reconstructed scene presents some holes because of the correlation errors - is considered as the input measure of a learning process, as was used for tracking of people in [4].

\subsection{Learning the surface of the organs}

The z-position (in the frame of the current position of the endoscope) of each point on the surface of the organs is individually modelled as a Gaussian distribution (mean and standard deviation) corrupted by z-values of foreground instruments moving in the operating field. The z-values obtained from each new stereo pair (when the correlationbased stereo was successful) are classified according to the 
current Z-statistics of the corresponding organ vertex. The $z$-value is integrated in the organ model only if it is either within the standard deviation of the corresponding model point, or further away from the observer. Hence, instruments that were inside the operating field at the beginning of the acquisition can be removed from the reconstruction. Everything that is not classified as part of the organ model is considered as belonging to the foreground. At each step, the classification map is filtered with morphological operators to correct the artifacts of segmentation.
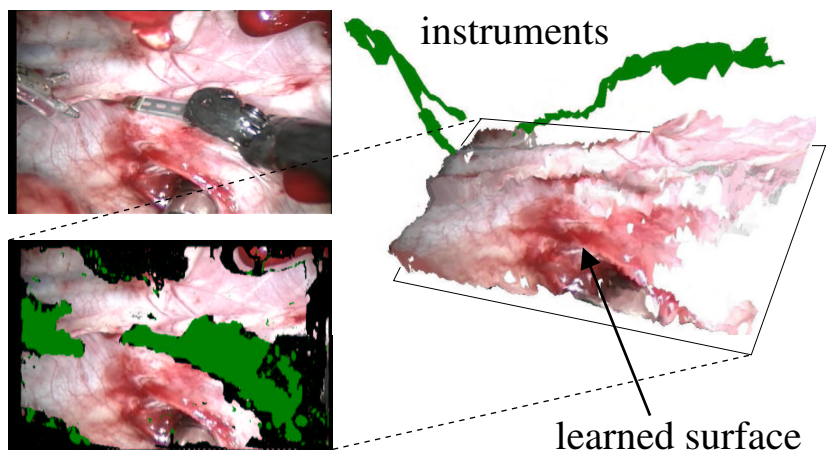

Figure 1. Top left: In vivo left view from the stereoscopic endoscope. Bottom left: result of the classification. Right: perspective view of the reconstruction showing the instruments above the surface of the organ learned from the previous images in the sequence.

\subsection{Removing the instruments from the operating field}

The learning of z-statistics of the surface of the organs provides the ability to continously classify pixels of new stereo pairs. Those not belonging to the organ model can be replaced by the last pixel value of the surface organ model leading to the masking of instruments. Moreover, this would enhance the integration of the coronary tree model in the endoscopic images by drawing arteries behind the instruments and view these through the surface of the organs.

\section{Results}

The first experiments were performed on image sequences of a plastic heart and on true per-operative sequences with the Cardiac Surgery team at Hôpital Européen Georges Pompidou, Paris, France using the da Vinci ${ }^{\mathrm{TM}}$ surgical system. Figure 1 shows a left endoscopic in vivo image and the result of the classification. The black pixels could not be classified because of the correlation errors in the stereoscopy algorithm or because of the poor quantity of useful data in the sequence (on the top centre of the image, the correlation often failed on a foreground moving tissue). The 3D scene shows the instant reconstruction of the tools on the learned surface of the organ in the background. There are some imperfections due to specular reflections, but the reconstruction of the instruments is not our main objective.

\section{Discussion and future trends}

We have presented a method for building a 3D model of the surface of the organs observed by a stereoscopic endoscope, despite the existence of instruments in the operating field. A more global reconstruction of the observed organ requires the fusion of small surface patches. We are working on the estimation of the endoscopic motion from stereo tracking of points of interest (if manually controlled) or from the robot's articular coordinates if the endoscope is robotized. The choice of an appropriate representation and parametrisation of the reconstructed surface would allow to reduce the complexity of the data fusion problem and to deal with the difficult problem of the organ's motion.

Then the registration of the surface of the organs with pre-operative segmentation results will introduce new realtime challenges. We hope to run this whole image processing loop at $1 \mathrm{~Hz}$, with a parallel implementation of the algorithms, but overlaying at video rate will require to compensate endoscope and organ motion.

\section{References}

[1] Y. Akatsuka, T. Kawamata, M. Fujii, Y. Furuhashi, T. Saito, A. amd Shibasaki, H. Iseki, and T. Hori. AR navigation system for neurourgery. In Proceedings MICCAI, volume 1935 of Lecture Notes in Computer Science, pages 833-838. Springer, Oct. 2000.

[2] J. Cortadellas, G. Bellaire, and G. Grasche. New concepts for intraoperative navigation: calibration of a 3-D laparoscope. In BVM'2000 - Bildverarbeitung für die Medizin, pages 158162, Munich, Germany, 2000. Springer Verlag.

[3] F. Devernay, F. Mourgues, and E. Coste-Manière. Towards endoscopic augmented reality for robotically assisted minimally invasive cardiac surgery. In Proceedings of Medical Imaging and Augmented Reality, 2001.

[4] C. Eveland, K. Konolige, and R. Bolles. Background modeling for segmentation of video-rate stereo sequences. CVPR, pages 266-272, 1998.

[5] H. Fuchs, M. Livingston, R. Raskar, D. Colucci, K. Keller, A. State, J. Crawford, P. Rademacher, S. Drake, and A.Meyer. Augmented reality visualization for laparoscopic surgery. In Proceedings MICCAI, volume 1496 of Lecture Notes in Computer Science, pages 934-943. Springer, 1998.

[6] D. Loulmet, A. Carpentier, N. d'Attellis, A. Berrebi, C. Cardon, O. Ponzio, B. Aupècle, and J. Y. M. Relland. Endoscopic coronary artery bypass grafting with the aid of robotic assisted instruments. The journal of thoracic and cardiovascular surgery, 118(1), July 1999.

[7] F. Mourgues, F. Devernay, G. Malandain, and E. CosteManière. $3 \mathrm{~d}+\mathrm{t}$ modeling of coronary artery tree from standard non simultaneous angiograms. In Proceedings MICCAI, Lecture Notes in Computer Science. Springer, 2001. To appear.

[8] Z. Zhang. Flexible camera calibration by viewing a plane from unknown orientations. In Proceedings of the 7th International Conference on Computer Vision, pages 666-673, Kerkyra, Greece, 1999. IEEE Computer Society, IEEE Computer Society Press. 